\title{
ON SOME EXPONENTIAL MEANS. PART II
}

\author{
JÓZSEF SÁNDOR AND GHEORGHE TOADER
}

Received 18 May 2005; Revised 19 April 2006; Accepted 21 June 2006

We prove some new inequalities involving an exponential mean, its complementary, and some means derived from known means by applying the exp-log method.

Copyright (c) 2006 Hindawi Publishing Corporation. All rights reserved.

\section{Introduction}

All the means that appear in this paper are functions $M: \mathbb{R}_{+}^{2} \rightarrow \mathbb{R}_{+}$with the property that

$$
\min (a, b) \leq M(a, b) \leq \max (a, b) \quad \forall a, b>0 .
$$

Of course $M(a, a)=a$, for all $a>0$. As usual $A, G, L, I, A_{p}$ denote the arithmetic, geometric, logarithmic, identric, respectively, power means of two positive numbers, defined by

$$
\begin{gathered}
A=A(a, b)=\frac{a+b}{2}, \quad G=G(a, b)=\sqrt{a b} \\
L=L(a, b)=\frac{b-a}{\log b-\log a}, \quad I=I(a, b)=\frac{1}{e}\left(\frac{b^{b}}{a^{a}}\right)^{1 /(b-a)}, \\
A_{p}=A_{p}(a, b)=\left(\frac{a^{p}+b^{p}}{2}\right)^{1 / p}, \quad p \neq 0 .
\end{gathered}
$$

In [16], the first part of this paper, we have studied the exponential mean

$$
E=E(a, b)=\frac{b e^{b}-a e^{a}}{e^{b}-e^{a}}-1
$$

introduced in [23]. Another exponential mean was defined in [19] by

$$
\bar{E}=\bar{E}(a, b)=\frac{a e^{b}-b e^{a}}{e^{b}-e^{a}}+1
$$

Hindawi Publishing Corporation International Journal of Mathematics and Mathematical Sciences Volume 2006, Article ID 51937, Pages 1-9

DOI 10.1155/IJMMS/2006/51937 
2 On some exponential means. Part II

It is the complementary of $E$, according to a definition from [4], that is,

$$
\bar{E}=2 A-E
$$

A basic inequality proved in $[23]$ is

$$
E>A \text {, }
$$

which gives the new inequality

$$
\bar{E}<A \text {. }
$$

More general means have been studied in $[14,17,19]$. For example, letting $f(x)=e^{x}$ in $[14$, Formula (5)], we recapture (1.6). We note that by selecting $f(x)=\log x$ in $[14$, Formula (8)], and then $f(x)=1 / x$, we get the standard inequalities

$$
G<L<I<A
$$

(for history, see, e.g., [7]).

In what follows, for any mean $M$, we will denote by $\mathcal{M}$ the new mean given by

$$
M(x, y)=\log M\left(e^{x}, e^{y}\right), \quad x, y>0 .
$$

As we put $a=e^{x}, b=e^{y}$ and then take logarithms, we call this procedure the exp-log method. The method will be applied also to some inequalities for deriving new inequalities. For example, in [16] we proved that

$$
E=\mathscr{I}
$$

and so (1.8) becomes

$$
A<\mathscr{L}<E<\mathscr{A}
$$

In [16], it was also shown that

$$
\begin{gathered}
A+\mathscr{A}-\mathscr{L}<E<2 \mathscr{L}-A, \\
\mathscr{A}_{2 / 3}<E<\mathscr{A}_{\log 2}
\end{gathered}
$$

(see also $[6,22])$. In [9], the first author improved the inequality (1.6) by

$$
E>\frac{A+2 \mathscr{A}}{3}>A \text {. }
$$

This is based on the following identity proved there:

$$
(E-A)(a, b)=\frac{A\left(e^{a}, e^{b}\right)}{L\left(e^{a}, e^{b}\right)}-1 .
$$


We get the same result using the known result

$$
I>\frac{2 A+G}{3}>\left(A^{2} G\right)^{1 / 3}
$$

and the exp-log method.

The aim of this paper is to obtain other inequalities related to the above means.

\section{Main results}

(1) After some computations, the inequality (1.6) becomes

$$
\frac{e^{b}-e^{a}}{b-a}<\frac{e^{a}+e^{b}}{2}
$$

This follows at once from the Hadamard inequality

$$
\frac{1}{b-a} \int_{a}^{b} f(t) d t<\frac{f(a)+f(b)}{2}
$$

applied to the strictly convex function $f(t)=e^{t}$. We note that by the second Hadamard inequality, namely

$$
\frac{1}{b-a} \int_{a}^{b} f(t) d t>f\left(\frac{a+b}{2}\right)
$$

for the same function, one obtains

$$
\frac{e^{b}-e^{a}}{b-a}>e^{(a+b) / 2}
$$

which has been proposed as a problem in [3].

The relation (1.11) improves the inequality (2.1), which means that $\mathscr{A}>\mathscr{L}$, and improves (2.4), which means that $\mathscr{L}>A$. In fact, by the above remarks, one can say that

$$
E>A \Longleftrightarrow \mathscr{A}>\mathscr{L} \text {. }
$$

(2) In [23], it was proven that $E$ is not comparable with $A_{\lambda}$ for $\lambda>5 / 3$. Then in [17], we have shown, among others, that

$$
A(a, b)<E(a, b)<A(a, b) \cdot e^{|b-a| / 2} .
$$

Now, if $|b-a|$ becomes small, clearly $e^{|b-a| / 2}$ approaches to 1 , that is, the conjecture $E>A_{\lambda}$ of [23] cannot be true for any $1<\lambda \leq 5 / 3$.

We get another double inequality from (1.5) and (1.6):

$$
A<E<2 A \text {. }
$$

These inequalities cannot be improved. Indeed, for $1<\lambda<2$, we have

$$
\lim _{x \rightarrow \infty}[E(1, x)-\lambda A(1, x)]=\infty,
$$


4 On some exponential means. Part II

but

$$
E(1,1)-\lambda A(1,1)=1-\lambda<0,
$$

thus $E$ is not comparable with $\lambda A$.

On the other hand,

$$
\bar{E}(a, b)=\frac{e^{b}(a+1)-e^{a}(b+1)}{e^{b}-e^{a}}=(a+1)(b+1) \cdot \frac{f(b)-f(a)}{e^{b}-e^{a}},
$$

where $f(x)=e^{x} /(x+1)$. By Cauchy's mean value theorem,

$$
\frac{f(b)-f(a)}{e^{b}-e^{a}}=\frac{f^{\prime}(c)}{e^{c}}, \quad c \in(a, b) .
$$

Since

$$
\frac{f^{\prime}(c)}{e^{c}}=\frac{c}{(c+1)^{2}} \leq \frac{1}{4}
$$

we get

$$
0<2 A-E \leq \frac{(a+1)(b+1)}{4} .
$$

(3) By using the series representation

$$
\log \frac{I}{G}=\sum_{k=1}^{\infty} \frac{1}{2 k+1}\left(\frac{b-a}{b+a}\right)^{2 k},
$$

(see $[9,21])$, we can deduce the following series representation:

$$
(E-A)(a, b)=\sum_{k=1}^{\infty} \frac{1}{2 k+1}\left(\frac{e^{b}-e^{a}}{e^{b}+e^{a}}\right)^{2 k}
$$

By (2.1), $\left|e^{b}-e^{a}\right| /\left(e^{b}+e^{a}\right)<|b-a| / 2$, thus we get the estimate

$$
(E-A)(a, b)<\sum_{k=1}^{\infty} \frac{1}{2 k+1}\left(\frac{b-a}{2}\right)^{2 k} .
$$

The series is convergent at least for $|b-a|<2$. Writing

$$
\frac{A\left(e^{a}, e^{b}\right)}{L\left(e^{a}, e^{b}\right)}=e^{\mathscr{A}(a, b)-\mathscr{L}(a, b)}
$$

the identity (1.14) implies the relation

$$
E-A=e^{\mathscr{A}-\mathscr{L}}-1
$$


This gives again the equivalence (2.5). But one can obtain also a stronger relation by writing $e^{x}>1+x+x^{2} / 2$, for $x>0$. Thus (2.18) gives

$$
E-A>\mathscr{A}-\mathscr{L}+\frac{1}{2}(\mathscr{A}-\mathscr{L})^{2} .
$$

(4) Consider the inequality proved in [10]:

$$
\frac{2}{e} A<I<A
$$

By the exp-log method, we deduce

$$
\log 2-1+\mathscr{A}<E<\mathscr{A}
$$

From the inequality

$$
I<\frac{2}{e}(A+G)=\frac{4}{e}\left(\frac{\sqrt{a}+\sqrt{b}}{2}\right)^{2},
$$

given in [5], we have, by the same method,

$$
E(x, y)<2 \log 2-1+2 \mathscr{A}\left(\frac{x}{2}, \frac{y}{2}\right)
$$

Relation (2.23) may be compared with the left-hand side of (2.21). Take now the relation

$$
L<L(A, G)=\frac{A-G}{\log (A / G)}
$$

from [5]. Since $A-G=1 / 2(\sqrt{a}-\sqrt{b})^{2}$, one obtains

$$
\mathscr{A}-A<\frac{1}{2 e^{\mathscr{L}}}\left(e^{x / 2}-e^{y / 2}\right)^{2} .
$$

The relation

$$
L^{3}>\left(\frac{A+G}{2}\right)^{2} G
$$

from [13], gives similarly

$$
3 \mathscr{L}(x, y)>A(x, y)+4 \mathscr{A}\left(\frac{x}{2}, \frac{y}{2}\right),
$$

while the inequality

$$
\log \frac{I}{L}>1-\frac{G}{L}
$$

from [7], offers the relation

$$
E-\mathscr{L}>1-e^{A-\mathscr{L}} .
$$


6 On some exponential means. Part II

(5) The exp-log method applied to the inequality

$$
L>\sqrt{G I}
$$

given in $[2,11]$, implies that

$$
\mathscr{L}>\frac{A+E}{2}>\frac{2 A+\mathscr{A}}{3} .
$$

On the other side, the inequality

$$
I>\sqrt{A L},
$$

proven in [11], gives on the same way the inequality

$$
E>\frac{\mathscr{A}+\mathscr{L}}{2} \text {. }
$$

After all, we have the double inequality

$$
\frac{\mathscr{A}+\mathscr{L}}{2}<E<2 \mathscr{L}-A .
$$

(6) Consider now the inequality

$$
3 I^{2}<2 A^{2}+G^{2},
$$

from [20]. It gives

$$
\log 3+2 E<\log \left(e^{2 A}+2 e^{2 \mathscr{A}}\right) .
$$

Similarly

$$
I>\frac{2 A+G}{3},
$$

given in [8], implies that

$$
\log 3+E>\log \left(2 e^{A}+e^{\mathscr{A}}\right) .
$$

In fact, the relation

$$
I>\frac{A+L}{2},
$$

from [7], gives

$$
\log 2+E>\log \left(e^{\mathscr{L}}+e^{\mathscr{A}}\right),
$$

but this is weaker than (2.38), as follows from [8]. The inequalities (2.33) and (2.40) can be combined as

$$
E>\log \left(\frac{e^{\mathscr{L}}+e^{\mathscr{A}}}{2}\right)>\frac{\mathscr{L}+\mathscr{A}}{2}
$$


where the second inequality is a consequence of the concavity of the logarithmic function. We notice also that by

$$
L+I<A+G
$$

given in [1], one can write

$$
e^{\mathscr{L}}+e^{E}<e^{\mathscr{A}}+e^{A}
$$

(7) In [9] Sándor proved the inequality

$$
I\left(a^{2}, b^{2}\right)<\frac{A^{4}(a, b)}{I^{2}(a, b)} .
$$

By the exp-log method, we get

$$
E(2 x, 2 y)<4 \mathscr{A}(x, y)-2 E(x, y) \text {. }
$$

It is interesting to note that by the equality

$$
\log \frac{I^{2}(\sqrt{a}, \sqrt{b})}{I(a, b)}=\frac{G(a, b)}{L(a, b)}-1,
$$

given in [7], we have the identity

$$
2 E\left(\frac{x}{2}, \frac{y}{2}\right)-E(x, y)=e^{A(x, y)-\mathscr{L}(x, y)}-1 .
$$

Putting $x \rightarrow x / 2, y \rightarrow y / 2$ in (2.45), and taking into account (2.47), we can write

$$
2 E(x, y)+e^{A(x, y)-\mathscr{L}(x, y)}-1<4 \mathscr{A}\left(\frac{x}{2}, \frac{y}{2}\right) .
$$

This may be compared to (2.23).

(8) We consider now applications of the special Gini mean

$$
S=S(a, b)=\left(a^{a} b^{b}\right)^{1 /(a+b)}
$$

(see [15]). Its attached mean (by the exp-log method)

$$
\mathscr{S}(x, y)=\frac{x e^{x}+y e^{y}}{e^{x}+e^{y}}=\log S\left(e^{x}, e^{y}\right)
$$

is a special case of

$$
M_{f}(x, y)=\frac{x f(x)+y f(y)}{f(x)+f(y)}
$$

which was defined in [18]. Using the inequality

$$
\left(\frac{S}{A}\right)^{2}<\left(\frac{I}{G}\right)^{3}
$$


from [15], we get

$$
2 \mathscr{S}-2 \mathscr{A}<3 E-3 A \text {. }
$$

The inequalities

$$
\frac{A^{2}}{I}<S<\frac{A^{4}}{I^{3}}<\frac{A^{2}}{G}
$$

given in [15] imply that

$$
2 \mathscr{A}-E<\mathscr{S}<4 \mathscr{A}-3 E<2 \mathscr{A}-A \text {. }
$$

These offer connections between the exponential means $E$ and $\mathscr{Y}$.

Let now the mean

$$
U=U(a, b)=\frac{1}{3} \sqrt{(2 a+b)(a+2 b)} .
$$

In [12], it is proved that

$$
G<\sqrt[4]{U^{3} G}<I<\frac{U^{2}}{A}<U<A
$$

By the exp-log method, we get

$$
A<\frac{1}{4}(3 \boldsymbol{U}+A)<E<2 \boldsymbol{U}-\mathscr{A}<\boldsymbol{U}<\mathscr{A} .
$$

These relations offer a connection between the means $E$ and $\mathcal{U}$.

\section{Acknowledgments}

The authors thank the two referees for useful remarks which have improved the presentation of the paper.

\section{References}

[1] H. Alzer, Ungleichungen für Mittelwerte, Archiv der Mathematik 47 (1986), no. 5, 422-426.

[2] __ Two inequalities for means, Comptes Rendus Mathématiques de L'Academie des Sciences. La Société Royale du Canada. (Mathematical Reports) 9 (1987), no. 1, 11-16.

[3] S.-J. Bang, Problem 4472, School Science and Mathematics 95 (1998), 222-223.

[4] C. Gini, Le Medie, Unione Tipografico Torinese, Milano, 1958.

[5] E. Neuman and J. Sándor, On certain means of two arguments and their extensions, International Journal of Mathematics and Mathematical Sciences 2003 (2003), no. 16, 981-993.

[6] A. O. Pittenger, Inequalities between arithmetic and logarithmic means, Univerzitet u Beogradu. Publikacije Elektrotehničkog Fakulteta. Serija Matematika i Fizika (1980), no. 678-715, 15-18 (1981).

[7] J. Sándor, On the identric and logarithmic means, Aequationes Mathematicae 40 (1990), no. 2-3, 261-270.

[8] __ A note on some inequalities for means, Archiv der Mathematik 56 (1991), no. 5, 471473. 
[9] _ On certain identities for means, Studia Universitatis Babeş-Bolyai. Mathematica 38 (1993), no. 4, 7-14.

[10] On certain inequalities for means, Journal of Mathematical Analysis and Applications 189 (1995), no. 2, 602-606.

[11]_ On refinements of certain inequalities for means, Archivum Mathematicum (Brno) 31 (1995), no. 4, 279-282.

[12] _ Two inequalities for means, International Journal of Mathematics and Mathematical Sciences 18 (1995), no. 3, 621-623.

[13] _ On certain inequalities for means. II, Journal of Mathematical Analysis and Applications 199 (1996), no. 2, 629-635.

[14] _ On means generated by derivatives of functions, International Journal of Mathematical Education in Science and Technology 28 (1997), no. 1, 146-148.

[15] J. Sándor and I. Raşa, Inequalities for certain means in two arguments, Nieuw Archief voor Wiskunde. Vierde Serie 15 (1997), no. 1-2, 51-55.

[16] J. Sándor and Gh. Toader, On some exponential means, Seminar on Mathematical Analysis (ClujNapoca, 1989-1990), Preprint, vol. 90, “Babeş-Bolyai” Univ., Cluj, 1990, pp. 35-40.

[17] __ Some general means, Czechoslovak Mathematical Journal 49(124) (1999), no. 1, 53-62.

[18] On means generated by two positive functions, Octogon Mathematical Magazine 19 (2002), no. 1, 70-73.

[19] _ Inequalities for general integral means, Journal of Inequalities in Pure and Applied Mathematics 7 (2006), no. 1, article 13.

[20] J. Sándor and T. Trif, Some new inequalities for means of two arguments, International Journal of Mathematics and Mathematical Sciences 25 (2001), no. 8, 525-532.

[21] H.-J. Seiffert, Comment to problem 1365, Mathematics Magazine 65 (1992), 356.

[22] K. B. Stolarsky, The power and generalized logarithmic means, The American Mathematical Monthly 87 (1980), no. 7, 545-548.

[23] Gh. Toader, An exponential mean, Seminar on Mathematical Analysis (Cluj-Napoca, 19871988), Preprint, vol. 88, Univ. “Babeş-Bolyai”, Cluj, 1988, pp. 51-54.

József Sándor: Department of Mathematics, Babes-Bolyai University,

3400 Cluj-Napoca, Romania

E-mail address: jsandor@math.ubbcluj.ro

Gheorghe Toader: Department of Mathematics, Technical University of Cluj-Napoca, 3400 Cluj-Napoca, Romania

E-mail address: gheorghe.toader@math.utcluj.ro 


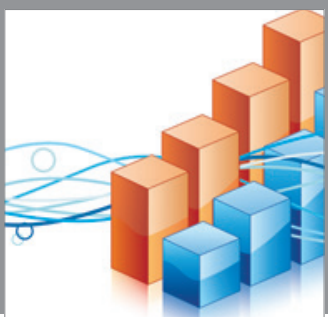

Advances in

Operations Research

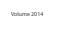

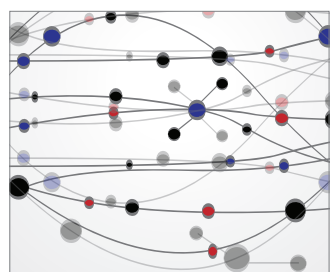

\section{The Scientific} World Journal
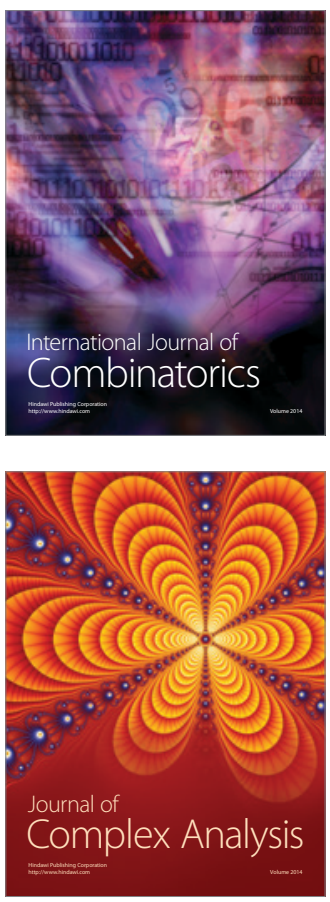

International Journal of

Mathematics and

Mathematical

Sciences
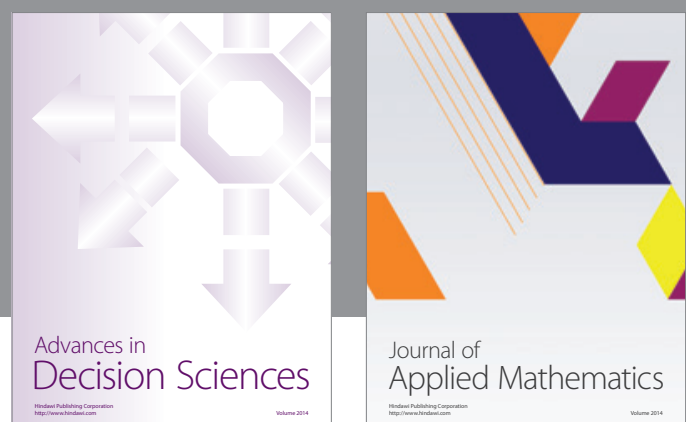

Journal of

Applied Mathematics
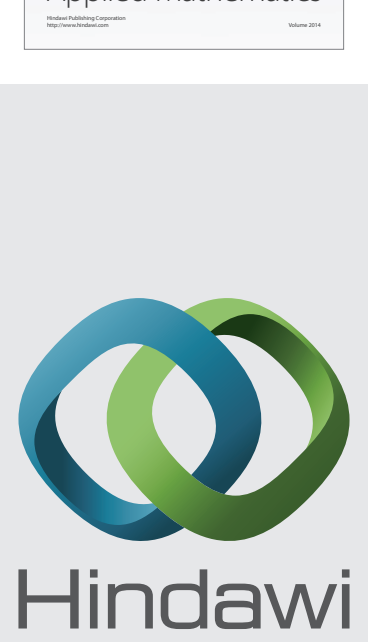

Submit your manuscripts at http://www.hindawi.com
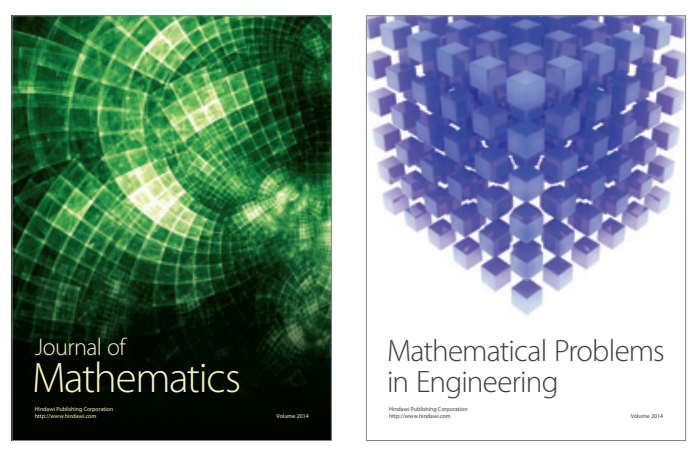

Mathematical Problems in Engineering
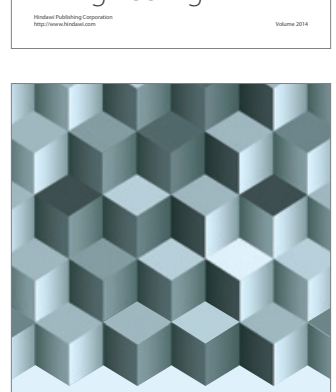

Journal of

Function Spaces
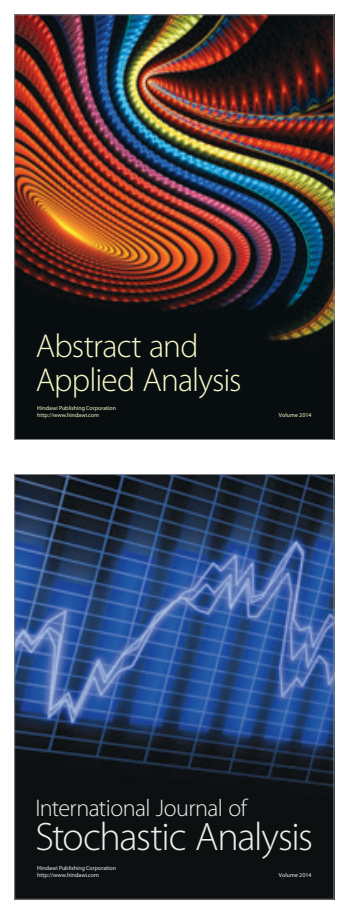

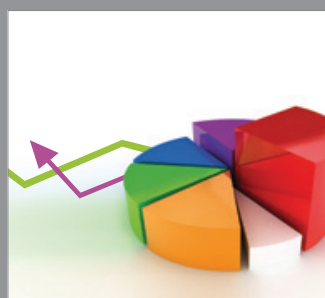

ournal of

Probability and Statistics

Promensencen
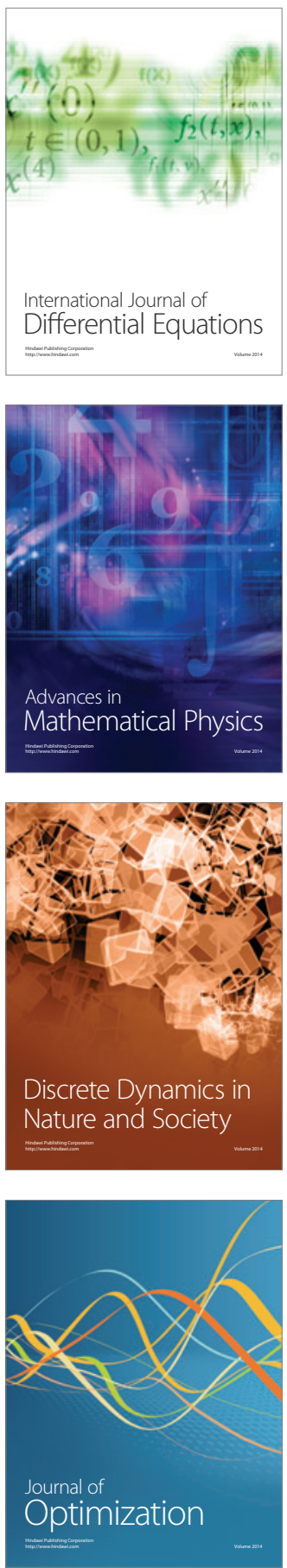